\title{
Impact of Mass Screening on the Number of Confirmed Cases, Recovered Cases, and Deaths Due to COVID-19 in Iran: An Interrupted Time Series Analysis
}

\author{
Moslem Taheri Soodejani, PhD'; Seyyed Mohammad Tabatabaei, PhD²,3 Ali Dehghani, PhD'; Willi McFarland, MD, PhD; Hamid Sharifi, \\ $\mathrm{PhD}^{5^{*}}$ \\ ${ }^{1}$ Research Center of Prevention and Epidemiology of Non-Communicable Disease, Department of Biostatistics and Epidemiology, Shahid \\ Sadoughi University of Medical Sciences, Yazd, Iran \\ ${ }^{2}$ Medical Informatics Department, School of Medicine, Mashhad University of Medical Sciences, Mashhad, Iran \\ ${ }^{3}$ Clinical Research Unit, Imam Reza Hospital, Mashhad University of Medical Sciences, Mashhad, Iran \\ ${ }^{4}$ Department of Epidemiology and Biostatistics, University of California San Francisco, San Francisco, CA, USA \\ ${ }^{5} \mathrm{HIV} / \mathrm{STI}$ Surveillance Research Center, and WHO Collaborating Center for HIV Surveillance, Institute for Futures Studies in Health, Kerman \\ University of Medical Sciences, Kerman, Iran
}

\begin{abstract}
Background: Mass screening for the coronavirus disease 2019 (COVID-19) began in Iran on March 23, 2020, with the purpose of improving early detection of patients for their own health and to prevent onward transmission to others. In this study, we evaluated the impact of the change towards mass screening on new cases reported, cases recovered, and deaths due to COVID-19.

Methods: This study analyzed the daily reports on the number of new cases confirmed by polymerase chain reaction (PCR) testing, cases recovered, and deaths due to COVID-19 provided to the Ministry of Health and Medical Education of Iran. Changes in trends on these outcomes were evaluated using interrupted time series analysis.

Results: From February 19 to May 6, 2020, a total of 519544 COVID-19 tests were done and 101650 diagnoses were made (case/ test ratio $19.6 \%$ ). For the same period, 6418 deaths due to COVID-19 were reported (case fatality ratio $6.3 \%$ ). The number of cases detected increased significantly over the period of scale-up of mass screening $(P=0.003)$, as did the number of recovered cases $(P=0.001)$. The number of deaths due to COVID-19 did not change before versus after mass screening.

Conclusion: Following the scale-up of mass screening for COVID-19 in Iran, the rate of new cases detected and reported recovered accelerated significantly. Mass screening is likely to have detected many mild and asymptomatic cases that were infectious. Our data support the role that mass screening, coupled with isolation and contract tracing, can have in slowing the COVID-19 epidemic.

Keywords: Coronavirus, Interrupted time series analysis, Screening, Severe acute respiratory syndrome related coronavirus 2, Pandemic

Cite this article as: Taheri Soodejani M, Tabatabaei SM, Dehghani A, McFarland W, Sharifi H. Impact of mass screening on the number of confirmed cases, recovered cases, and deaths due to COVID-19 in Iran: an interrupted time series analysis. Arch Iran Med. 2020;23(11):776-781. doi: 10.34172/aim.2020.103.
\end{abstract}

Received: June 4, 2020, Accepted: September 6, 2020, ePublished: November 1, 2020

\section{Introduction}

The coronavirus disease 2019 (COVID-19) pandemic started at the end of 2019 in Wuhan, China, rapidly spread worldwide, and has affected all aspects of life. SARS-COV-2, the virus that causes COVID-19 disease, is arguably the world's foremost health challenge at this moment. The virus has infected millions and killed hundreds of thousands of people in the span of a few months. ${ }^{1,2}$ The disease has also hurt economies, produced great sadness for those who have lost loved ones, interrupted the education of children and young adults, and severely affected social relationships. Iran has been one of the most severely affected countries, with the highest number of reported infected cases and deaths in the East Mediterranean Region. ${ }^{3}$ Fortunately, the number of recovered cases has increased over time. ${ }^{1}$

Early detection, with isolation and contact tracing, are tools to slow the spread of infection for contagious diseases. These activities can reduce the basic reproductive number $\left(\mathrm{R}_{0}\right)$ and effective reproductive number $\left(\mathrm{R}_{\mathrm{e}}\right)$ to drive new infections to zero. Mass screening can increase early detection through large-scale testing of persons in communities regardless of symptoms. Mass screening can identify otherwise undetected cases and has been applied to the control and mitigation other diseases. ${ }^{4-7}$

Beginning on March 23, 2020, one month after the first confirmed case in the country, Iran adopted a mass screening strategy as a measure to better manage the 
COVID-19 epidemic. The impact of this mass screening has not been previously investigated. This study, therefore, aims to assess the impact of mass screening on changing trends in the numbers of confirmed cases, cases who have recovered, and deaths due to COVID-19 using reported surveillance data and interrupted time series analysis.

\section{Materials and Methods}

Data

Outcome measures were cases reported daily to the Ministry of Health and Medical Education (MOHME) from February 19 through May 6, 2020. We modeled the number of reported COVID-19 cases confirmed by positive polymerase chain reaction (PCR) test, the number recovered from infection by 14 days after resolution of symptoms and a negative PCR test result, and the number of reported deaths due to COVID-19. These data are available to the public on the MOHME website ${ }^{8}$ and from the World Health Organization (WHO). ${ }^{3}$

\section{Mass Screening for COVID-19}

At the onset of the epidemic in Iran February 19, 2020, testing and surveillance for COVID-19 were passive, with cases reported from health centers where individuals were treated. After March 23, 2020, mass screening by COVID-19 PCR testing and CT scan of the chest was scaled up throughout the country. Case reporting thereafter occurred through several avenues, including completion of an online self-declaration form, health authorities making phone calls to people in the community, and contact tracing. People with symptoms of COVID-19 were referred to the nearest designated center for further examination and diagnostic testing. In combination, these methods led to the screening of more than 70 million people, which is over $90 \%$ of the Iranian population. ${ }^{9}$

\section{Interrupted Time Series Analysis}

Interrupted time series analysis was used to evaluate the effect of mass screening on reporting of three outcomes: confirmed cases, recovered cases, and deaths due to the COVID-19. The model includes four parameters. Parameter $\beta_{0}$ represents the baseline level of the outcome at beginning of the epidemic (time $=0$ ). Parameter $\beta_{1}$ is the change in outcome associated with each unit of time (slope) for the pre-mass screening period. Parameter $\beta_{2}$ is the level change in the outcome for the pre- versus postmass screening intervention. Parameter $\beta_{3}$ indicates the change in trend (change in slope) of the outcome following the intervention (using the interaction between time and intervention level). Formula 1 below shows the model.

Formula 1: outcome $=\beta_{0}+\beta_{1}($ time $)+\beta_{2}($ level $)+\beta 3($ time $x$ level) $+\varepsilon$

The correlation between time and outcome variables (confirmed cases, recovered cases, and deaths) was statistically significant $(P=0.001)$. Diagnostic plots were used to check linear assumptions. According to the plot of residuals versus fitted values, no pattern was detected; therefore, linear models were applied. We further examined the increase or decrease in observed cases, recovered cases, and deaths over the expected number in the first six weeks following mass screening. Before running the final model, the Durbin-Watson statistic was used to detect autocorrelation. The Durbin-Watson statistics in the $6^{\text {th }}$ lag for confirmed cases, recovered cases, and deaths were $1.53(P=0.19), 1.58(P=0.14)$ and $1.49(P=0.07)$, respectively.

\section{Results}

Through May 6, 2020, a total of 519544 diagnostic COVID-19 PCR tests were performed in Iran. Overall, 101650 cases were confirmed (case/test ratio 19.6\%). During this period, 81578 confirmed cases recovered and 6418 died (case fatality ratio 6.3\%); 13654 (13.4\%) were hospitalized with unknown outcome.

Figure 1 and Table 1 describe the trends in confirmed COVID-19 case finding. From baseline $\left(\beta_{0}\right)$, case finding was significantly increasing over the period before the mass screening effort $\left(\beta_{1} 1141.5,95 \%\right.$ CI 822.2, 1460.8). After mass screening on $23 \mathrm{March}, 2002$, the level of case finding did not immediately change significantly $\left(\beta_{2}-324.6,95 \%\right.$ CI -669.2, 19.9). However, case detection accelerated with the scale-up of mass testing thereafter $\left(\beta_{3} 663.2,95 \% \mathrm{CI}\right.$ : 230.8, 1095.6).

Figure 2 shows the trends in reported cases recovered from COVID-19, with parameters reported in Table 1. The data indicate no significant change in the number of reported cases recovered at baseline, over time during the pre-mass screening period, or immediately following the mass screening period. The number of reported recovered cases accelerated significantly after mass screening $\left(\beta_{3}\right.$ 1626, 95\% CI 1270.3, 1981.7).

With respect to reported deaths due to COVID-19 (Figure 3 and Table 1), the pre-mass screening period shows a significant increase in the rate of reported deaths over time $\left(\beta_{1} 109.7,95 \%\right.$ CI 90.3, 129.1). No significant change was seen in the number of reported deaths immediately pre- versus post-mass screening $\left(\beta_{2} 6.9\right.$, 95\% CI, -12.7, 26.5). The number of deaths reported due to COVID-19 after mass screening decelerated, but not significantly $\left(\beta_{3}-22.1,95 \%\right.$ CI $\left.-46.6,2.4\right)$.

Changes in the number and percentage of confirmed cases, recovered cases, and deaths were investigated at the end of six weeks after the mass screening (Table 2). As a differential from the expected (baseline or counterfactual), the observed results were an increase by $27.0 \%$ in confirmed cases, $85.4 \%$ in cases recovered, and a decrease of $13.4 \%$ in deaths. The case/fatality ratio of COVID-19 before and after mass screening was 7.8\% (95\% CI 7.5, 


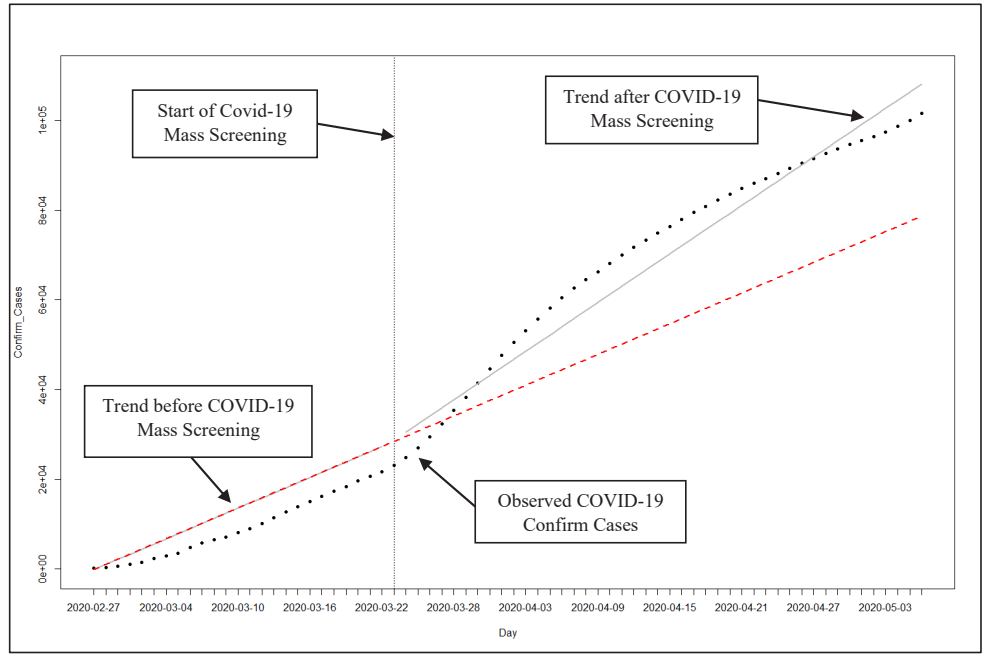

Figure 1. Cases with Confirmed COVID-19 in Iran before and after Mass Screening. Vertical line indicates the start of mass screening; dotted line indicates observed confirmed COVID-19 cases; dashed line indicates trend before mass screening; solid line indicates trend after mass screening.

$8.2 \%)$ and $5.8 \%(95 \%$ CI 5.7, 6.0\%), respectively.

\section{Discussion}

Our study provides empirical support to the potential impact that mass screening can have on slowing the COVID-19 epidemic. With scale-up of mass screening for COVID-19 in Iran, the number of cases detected and reported to the MOHME accelerated substantially compared to the baseline period of passive detection and reporting. Detecting the highest number of persons with infection as early as possible can guide and improve control efforts such as isolation, contact tracing, and targeting of further testing. ${ }^{5-7} \mathrm{~A}$ mass screening approach with isolation of cases may be the most effective means to control diseases for which there is no vaccine or effective treatment, such as COVID-19 at present. Testing patients regardless of symptoms also addresses transmission from asymptomatic persons..$^{10}$ Mass screening, in conjunction with restricted movement and activities, was a strategy for COVID-19 control in Wuhan, China. The approach is believed to have prevented substantial transmission in the city and blunted secondary peaks ${ }^{11}$ In the United Kingdom, mass testing with isolation of cases projects substantial reduction in the prevalence of the disease over time. ${ }^{12} \mathrm{WHO}$ guidelines currently encourage countries to improve case detection, implement contact tracing, isolate cases, and quarantine their close contacts. ${ }^{13}$

We also observed an acceleration in the reporting of cases recovered from COVID-19 following the scale-up of mass screening compared to baseline. Two explanations for this finding are possible. First, without mass screening, many COVID-19 cases, even those who were symptomatic, would have been undiagnosed and unreported. Therefore, their recovery from illness would also have been unreported. Second, mass screening would also diagnose mild and asymptomatic cases who would not have come to medical attention. Mass screening, which is performed at the community level, leads to identifying hidden, less

Table 1. Interrupted Time Series Parameters to Evaluate Trends in Confirmed Cases, Recovered Cases, and Deaths Due to COVID-19 in Iran

\begin{tabular}{|c|c|c|c|}
\hline Variable & & Coefficient $(95 \% \mathrm{Cl})$ & $P$ Value \\
\hline \multirow{4}{*}{ Confirmed cases* } & Intercept $\left(\beta_{0}\right)$ & $-1297.4(-7623.7,50.28 .9)$ & 0.7 \\
\hline & Trend before mass screening $\left(\beta_{1}\right)$ & $1141.5(822.2,1460.8)$ & 0.001 \\
\hline & Level change immediately pre- vs post- mass screening $\left(\beta_{2}\right)$ & $-324.6(-669.2,19.9)$ & 0.07 \\
\hline & Change in trend after mass screening $\left(\beta_{3}\right)$ & $663.2(230.8,1095.6)$ & 0.003 \\
\hline \multirow{4}{*}{ Recovered cases** } & Intercept $\left(\beta_{0}\right)$ & $19.3(-4583.7,4622.4)$ & 0.99 \\
\hline & Trend before mass screening $\left(\beta_{1}\right)$ & $173.4(-88.5,435.3)$ & 0.2 \\
\hline & Level change immediately pre- vs post- mass screening $\left(\beta_{2}\right)$ & $-975.8(-2156.3,204.7)$ & 0.11 \\
\hline & Change in trend after mass screening $\left(\beta_{3}\right)$ & $1626(1270.3,1981.7))$ & 0.001 \\
\hline \multirow{4}{*}{ Deaths*** } & Intercept $\left(\beta_{0}\right)$ & $-382.4(-851.4,86.6)$ & 0.11 \\
\hline & Trend before mass screening $\left(\beta_{1}\right)$ & $109.7(90.3,129.1)$ & 0.001 \\
\hline & Level change immediately pre- vs post- mass screening $\left(\beta_{2}\right)$ & $6.9(-12.7,26.5)$ & 0.48 \\
\hline & Change in trend after mass screening $\left(\beta_{3}\right)$ & $-22.1(-46.6,2.4)$ & 0.08 \\
\hline
\end{tabular}

*Adjusted R square 0.98; **Adjusted R square 0.99; ***Adjusted R square 0.99 . 


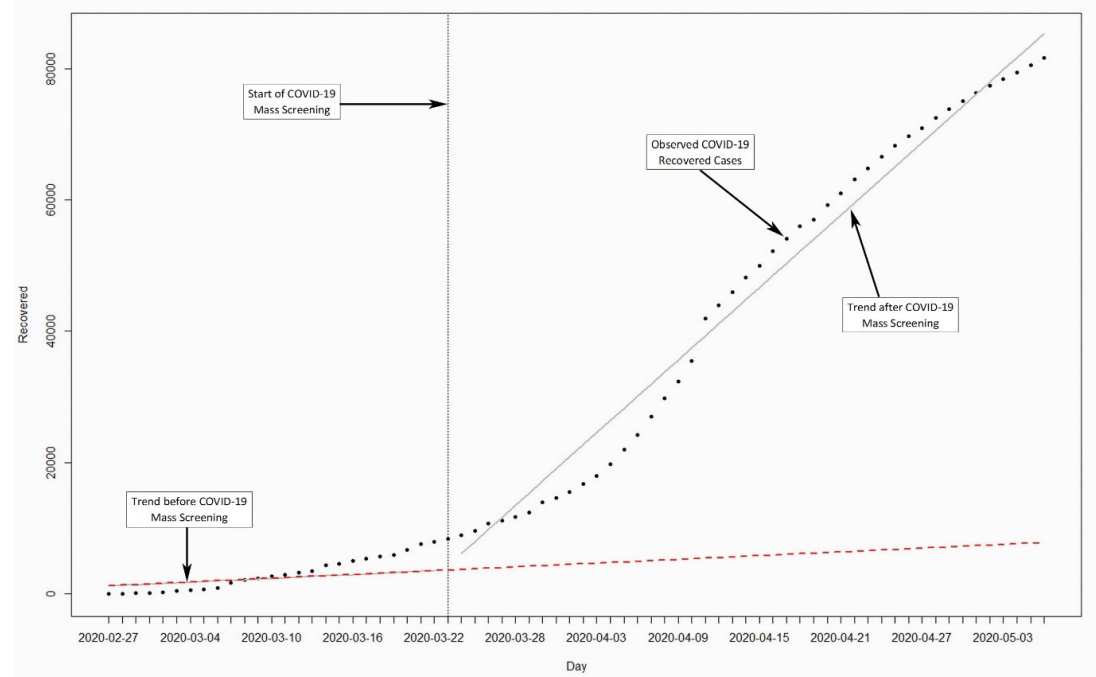

Figure 2. Cases Recovered from COVID-19 in Iran before and after Mass Screening. Vertical line indicates the start of mass screening; dotted line indicates cases recovered from COVID-19; dashed line indicates trend before mass screening; solid line indicates trend after mass screening.

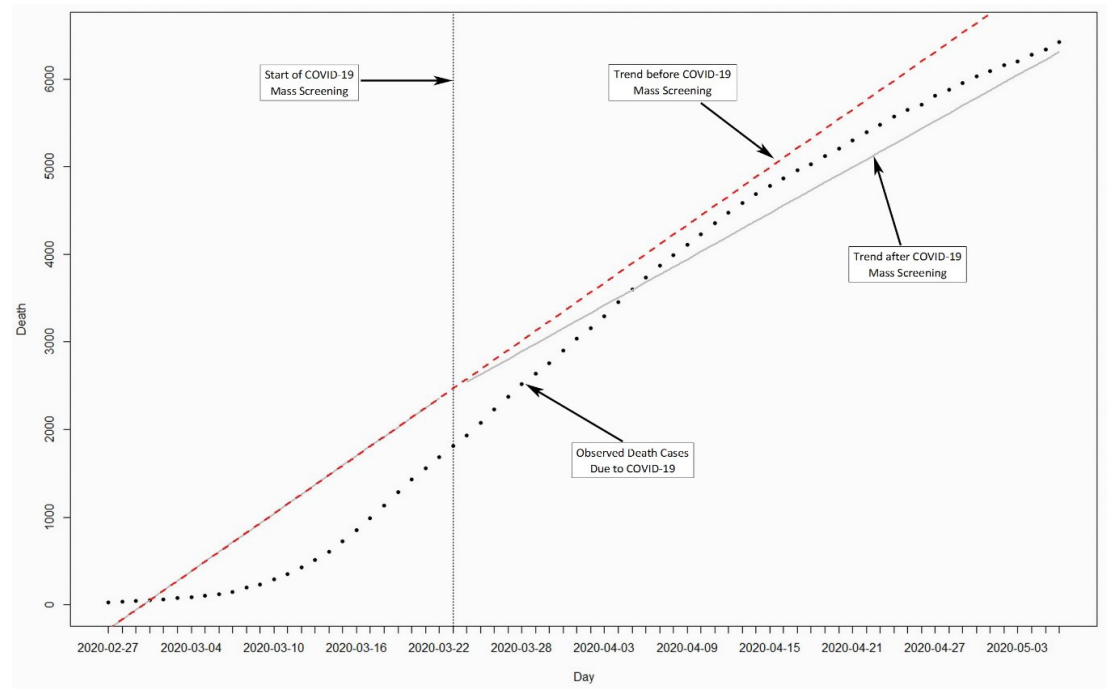

Figure 3. Deaths due to COVID-19 in Iran before and after Mass Screening. Vertical line indicates the start of mass screening; dotted line indicates deaths due to COVID-19; dashed line indicates trend before mass screening; solid line indicates trend after mass screening.

severe cases of the disease. ${ }^{14}$ Tracking the number of cases recovered from COVID-19 may have implications for reopening of economic and social activities as we learn more about immunity to infection. Mass screening has been suggested as a key part of the pathway back to "normal life", both for its ability to detect asymptomatic and mild cases and for identifying persons who may return to work.

We did not observe a significant increase in reported COVID-19-related deaths immediately after mass screening or accelerating over time. Although testing and diagnosis may influence completeness of reporting COVID-19 as a cause of death, the early diagnosis of mass screening did not appear to influence the ultimate course of the disease. The data did not suggest a change in the level of reported COVID-19 deaths immediately after mass screening. While there was a slight deceleration in the number of deaths in the post-mass screening period, this finding was not statistically significant.

We note indirect benefits of the mass screening program. In addition to identifying contagious cases with mild or no symptoms, the screening program provided training and educational opportunities. The program entailed health care workers reaching many persons by phone and in person. The contacted persons included many at highrisk for severe complications of COVID-19 disease, such as the elderly and people with underlying diseases. These interactions presented opportunities to educate people on the measures to take to protect themselves and others from infection. Educational activities were also done in Wuhan, China to control COVID-19. Results there showed a 
Table 2. Changes in the Number and Percentage of Confirmed Cases, Recovered Cases, and Deaths due to COVID-19 at the End of Each Week after Mass Screening in Iran.

\begin{tabular}{|c|c|c|c|c|c|c|}
\hline \multirow{2}{*}{$\begin{array}{l}\text { Week after } \\
\text { Mass Screening }\end{array}$} & \multicolumn{2}{|c|}{ Confirmed Cases $(95 \% \mathrm{Cl})^{*}$} & \multicolumn{2}{|c|}{ Recovered Cases $(95 \% \mathrm{CI})^{*}$} & \multicolumn{2}{|c|}{ Deaths $(95 \% \mathrm{Cl})^{*}$} \\
\hline & Count & $\%$ Change & Count & $\%$ Change & Count & $\%$ Change \\
\hline End of $1^{\text {st }}$ week & $4980(4856,5146)$ & $12.0(11.7,12.4)$ & $12025(11883,12167)$ & $67.7(66.9,68.5)$ & $-169(-168,-169)$ & $-5.25(-5.24,-5.26)$ \\
\hline End of $2^{\text {nd }}$ week & $9621(9459,9783)$ & $17.8(17.5,18.1)$ & $23400(23248,23552)$ & $77.1(76.6,77.6)$ & $-323(-322,-324)$ & $-8.12(-8.11,-8.12)$ \\
\hline End of $3^{\text {rd }}$ week & $14262(14062,14462)$ & $21.4(21.1,21.7)$ & $34775(34603,34903)$ & $81.0(80.6,81.3)$ & $-477(-476,-478)$ & $-10.06(-10.05,-10.07)$ \\
\hline End of $4^{\text {th }}$ week & $18903(18587,19140)$ & $23.9(23.5,24.2)$ & $46150(45983,46316)$ & $83.1(82.8,83.4)$ & $-631(-630,-632)$ & $-11.46(-11.45,-11.47)$ \\
\hline End of $5^{\text {th }}$ week & $23544(23268,23820)$ & $25.6(25.3,25.9)$ & $57525(57321,57661)$ & $84.5(84.2,84.7)$ & $-785(-784,-786)$ & $-12.52(-12.51,-12.53)$ \\
\hline End of $6^{\text {th }}$ week & $28185(27872,28498)$ & $27.0(26.7,27.3)$ & $68900(68739,69061)$ & $85.4(85.2,85.6)$ & $-939(-938,-940)$ & $-13.35(-13.34,-13.36)$ \\
\hline
\end{tabular}

$* 95 \%$ Confidence Interval.

The percent change is the observed (post-mass screening) minus the expected (pre-mass screening) over observed.

decrease in the number of severe cases and deaths and a reduction in the reproductive number $(\mathrm{R})$ from 3 to $1 .^{15}$ Similar training of health care providers and the general population on preventive measures, building on the experience with SARS in 2003, may have contributed to Taiwan's success against COVID-19. ${ }^{16}$

\section{Limitations}

We recognize that our data and analysis cannot prove that the mass screening measures caused reductions in the COVID-19. In addition to mass screening, with its accompanying education and training of individuals, other factors may have contributed. Closure of schools, universities, religious centers, and commercial areas, as well as implementation of social distancing are likely to have impacted the course of the COVID-19 epidemic in Iran.

\section{Conclusion}

Due to the fact that many with COVID-19 are asymptomatic, have mild symptoms, or do not come to medical attention yet still can transmit infection, it is imperative to identify these people to break the chains of transmission. Mass testing, with active case detection through contact tracing and isolation of cases, can ultimately help the country reduce morbidity and mortality due to COVID-19. The fact that new case detection and recovery accelerated over baseline while deaths did not, indicates that many persons with no or mild symptoms were detected as a result of mass testing. These persons may have otherwise never come to medical attention or implemented measures to protect others. The experience in Iran provides other countries with evidence of the potential role that mass testing may have on slowing their epidemic trajectories.

\section{Authors' Contribution}

MTS, AD, and HS designed the study. MTS conducted the primary analysis with guidance from MT, WM, and HS. MTS drafted the manuscript. WM edited the manuscript. All authors read, commented, and approve the final version.

\section{Conflict of Interest Disclosures} None.

\section{Ethical Statement}

As the data in this study were obtained from officially reported administrative data, no written, or oral consent form was required.

\section{References}

1. Coronavirus (COVID-19). World Heath Organization; 2020. Available from: https://who.sprinklr.com/. Accessed May 12, 2020.

2. Sohrabi C, Alsafi Z, O'Neill N, Khan M, Kerwan A, Al-Jabir A, et al. World Health Organization declares global emergency: A review of the 2019 novel coronavirus (COVID-19). Int J Surg. 2020;76:71-76. doi: 10.1016/j.ijsu.2020.02.034.

3. WHO Coronavirus Disease (COVID-19) Dashboard: World Health Organization; 2020. Available from: https://covid19. who.int/.

4. Soodejani MT, Mirzaei H, Manesh MM, Tabatabaei SM, Ghaderi A. Incidence of Colorectal Cancer and Adenomatous Polyps After a Two-Step Screening in Isfahan Province, Iran in 2018. J Gastrointest Cancer. 2020;51(3):850-854. doi: 10.1007/s12029-019-00313-x.

5. Paião DSG, Lemos EF, Carbone AdSS, Sgarbi RVE, Junior AL, da Silva FM, et al. Impact of mass-screening on tuberculosis incidence in a prospective cohort of Brazilian prisoners. BMC Infect Dis. 2016;16(1):533. doi: 10.1186/s12879-016-1868-5.

6. Hodgins S, Peeling R, Dery S, Bernier F, LaBrecque A, Proulx $J$, et al. The value of mass screening for chlamydia control in high prevalence communities. Sex Transm Infect. 2002;78 Suppl 1(Suppl 1):i64-8. doi: 10.1136/sti.78.suppl_1.i64.

7. Selent MU, Molinari NM, Baxter A, Nguyen AV, Siegelson $\mathrm{H}$, Brown $\mathrm{CM}$, et al. Mass screening for fever in children: a comparison of 3 infrared thermal detection systems. Pediatr Emerg Care. 2013;29(3):305-13. doi: 10.1097/ PEC.0b013e3182854465.

8. Factshhets (Persian): Ministry of Health and Medical Education; 2020. Available from: http://corona.behdasht.gov.ir/.

9. Factshhet16 (Persian): Ministry of Health and Medical Education; 2020. Available from: http://corona.behdasht.gov. ir/.

10. Hellewell J, Abbott S, Gimma A, Bosse NI, Jarvis Cl, Russell TW, et al. Feasibility of controlling COVID-19 outbreaks by isolation of cases and contacts. Lancet Glob Health. 2020;8(4):e488-e496. doi: 10.1016/S2214-109X(20)30074-7.

11. Prem K, Liu Y, Russell TW, Kucharski AJ, Eggo RM, Davies N, et al. The effect of control strategies to reduce social mixing on outcomes of the COVID-19 epidemic in Wuhan, China: a modelling study. Lancet Public Health. 2020;5(5):e261-e270. 
doi: 10.1016/S2468-2667(20)30073-6.

12. Peto J. Covid-19 mass testing facilities could end the epidemic rapidly. BMJ. 2020;368:m1163. doi: 10.1136/bmj.m1163.

13. World Health Organization. Critical preparedness, readiness and response actions for COVID-19: interim guidance, 22 March 2020. World Health Organization; 2020.

14. Nishimura Y, Tajima G, Bahagia AD, Sakamoto A, Ono $H$, Sakura N, et al. Differential diagnosis of neonatal mild hypergalactosaemia detected by mass screening: clinical significance of portal vein imaging. J Inherit Metab Dis.
2004;27(1):11-8. doi: 10.1023/B:BOLI.0000016621.29854. d6.

15. Pan A, Liu L, Wang C, Guo H, Hao X, Wang Q, et al. Association of public health interventions with the epidemiology of the COVID-19 outbreak in Wuhan, China. JAMA. 2020;323(19):1915-1923. doi: 10.1001/jama.2020.6130.

16. Wang CJ, Ng CY, Brook RH. Response to COVID-19 in Taiwan: big data analytics, new technology, and proactive testing. JAMA. 2020;323(14):1341-1342. doi: 10.1001/ jama.2020.3151. 